\title{
Effects of Dihydrostreptomycin Treatment on the Growth of Escherichia coli after Removal of Extracellular Antibiotic
}

\author{
By MARGOT KOGUT, J. W. LIGHTBOWN AND P. ISAACSON \\ National Institute for Medical Research, Mill Hill, London, N.W. 7
}

(Received 16 October 1964)

\begin{abstract}
SUMMARY
The growth characteristics of Escherichia coli B cultures treated with dihydrostreptomycin and then freed from extracellular antibiotic before growth had completely stopped, were examined. Growth, measured by extinction, proceeded exponentially, but at slower rates for a time, followed by gradual recovery. The degree of slowing of growth rate was a function of the duration of growth in the presence of a given concentration of dihydrostreptomycin. Comparison of viable colony count data and microscopic observation of such treated cultures showed that the majority of individuals in the populations must grow at the lower rates for two-three generations, after which some organisms cease to multiply and the rest recover. The proportion of organisms in treated populations which eventually ceased to grow was also a function of the duration of treatment. The amount of growth (cell synthesis), which had occurred at the time when onset of recovery became measurable, varied inversely with the $\%$ inhibition of growth rate. This suggests that recovery was due to some process not inhibited during the phase of inhibited exponential growth. It is concluded that intracellular dihydrostreptomycin consists of an 'inhibitory fraction' at the sites of inhibition, and a non-inhibitory 'pool' fraction; that the size of the latter varies between different individuals within a population and that transfer from 'pool' to inhibitory sites occurs by a process other than equilibration; e.g. that the factors which govern the uptake into these two phases must be, at least partly, independent. It is suggested that the degree of inhibition of growth rate reflects the extent of combination between antibiotic and inhibition sites at the time when extracellular dihydrostreptomycin is removed and no further uptake into the organisms can occur, and that the complex between dihydrostreptomycin and inhibition sites cannot dissociate to give active antibiotic which could re-enter the 'pool'.
\end{abstract}

\section{INTRODUCTION}

After an extensive survey of the literature for a review on the mode of action of streptomycin (Kogut \& Lightbown, 1964a), we became impressed with the desirability of dissociating the effects of this antibiotic on microbial growth from those connected with its entry into the organisms. Some aspects of the latter process have been described for a number of organisms by Pramer (1956), Litwak \& Pramer (1957), Szybalski \& Mashima (1959), Hancock (1962a) and Hurwitz \& Rosano (1962). In the preceding paper (Kogut, Lightbown \& Isaacson, 1965) we described a technique for studying the effects of dihydrostreptomycin on the growth of Escherichia coli B, uncomplicated by the continued accumulation of the antibiotic 
inside the organisms. The present paper deals with the characteristics of growth inhibition by dihydrostreptomycin under these conditions.

\section{METHODS}

The organism, medium, growth conditions, measurement of growth, preparation of inocula, dihydrostreptomycin treatment and procedure for removing extracellular dihydrostreptomycin, were as described by Kogut et al. (1965).

Colony counts were made by diluting appropriate samples in sterile growth medium without glucose, and plating on complete growth medium solidified with $1 \%(w / v)$ agar. Pour-plate and, in some experiments, drop counts (Miles \& Misra, 1938) were made, each colony count determination being obtained from platings in triplicate from each of two different dilutions. Colonies were counted after incubation at $33-35^{\circ}$ for $48-72 \mathrm{hr}$.

Direct observation of multiplication. For direct observation of the growth and division of bacteria from a culture, two methods of cultivating 20-60 organisms on solid media were used. Method A allowed the initial stage of growth, soon after the removal of extracellular dihydrostreptomycin, to be followed for about three divisions; method $\mathrm{B}$ was suitable for the observation of later phases of microcolony formation.

Method $A$. Samples withdrawn from the culture at the same time as plating for colony counts, were diluted in growth medium $+0.2 \%(\mathrm{w} / \mathrm{v})$ agar held at $35-40^{\circ}$, so that a drop which would just fill a Thoma counting chamber of $0.02 \mathrm{~mm}$. depth contained 30-60 organisms. A cover-slip was placed over the drop but not sealed, and the slide immediately placed on the stage of a Cooke, Troughton \& Simms binocular microscope which was mounted in a hot box, constructed of Perspex, with a thermostat and heater which allowed the ambient temperature to be held at $33-35^{\circ}$. The organisms were viewed by phase contrast with a $\times 40$ objective and a $\times 8$ ocular, and the position of the organisms in the grid of the Thoma chamber noted. The whole grid was then viewed at about hourly intervals and the numbers and positions of organisms recorded. The success of this method depended on the bacteria retaining their positions in the grid in which they were originally recorded. Usually, the agar set sufficiently for the movement of organisms to cease within 30-60 min. from the time they were placed on the slide. Preparations in which more than a few of the bacteria moved from their original positions after this time had to be discarded. Under these conditions it was possible to count with certainty up to ten organisms derived from individual bacteria at the first viewing, and to estimate up to about sixteen organisms per cluster. This technique allowed the fate of individual bacteria to be observed for about three generations. At later stages, the bacteria became rather crowded and may have exhausted their nutrients. Also, because of the longer generation times in dihydrostreptomycin-treated cultures, incubation beyond this point tended to produce drying of the agar. Postgate, Crumpton \& Hunter (1961) have described a similar method for the estimation of viability in a number of organisms. They found that the proportion of bacteria which did not give rise to visible colonies on conventional plate counts was the same as the proportion which did not divide at all during direct microscopic observation for 2-6 $\mathrm{hr}$, according to the organism and the growth temperature; this was approxi- 
mately three generation times. They used 'home-brewed tryptic meat medium' and state that the method was not suitable for establishing this relationship in the case of Escherichia coli в because these bacteria tended to form long ramifying filaments. Like Bretz (1962) we did not observe such filament formation under our conditions of growth on a mineral salts medium $+0.2 \%$ glucose and incubation at $33-35^{\circ}$.

Method B. In this method, for which we are indebted to Mr M. Young of this Institute, bacteria were cultivated on the surface of agar medium in such a way that they were evenly spread over a fairly large area and could be observed for $24 \mathrm{hr}$ or more. An 'England Finder' slide (Graticules Ltd., London, E.C. 1) was used; this has an etched grid of letters and numbers which can be viewed through a thin agar layer, thus allowing individual bacteria to be repeatedly located. The slide was placed on a levelling table and covered with a thin even layer of $1 \%$ agar nutrient medium by rapidly pipetting on to it $0.5 \mathrm{ml}$. which had been kept at $80-100^{\circ}$. After this had set (usually in 3-5 min.) it was trimmed with a razor to an area which was a little less than that of a 0.75 in. cover-slip. The slide was then kept in a sterile Petri dish containing moist lint until ready for use. Culture samples, withdrawn at the times of sampling for colony counting, and diluted in growth medium without glucose, were adjusted to concentrations of about 10,000 to 20,000 bacteria/ml. A drop of this suspension (about $0.01 \mathrm{ml}$.) was placed on the agar square and spread slightly by tilting. When the drop was the right size, and a cover-slip placed carefully on it, the bacteria were evenly distributed. When the drop was too large the placing of the cover-glass tended to crowd the organisms at the edges of the agar square. A seal of liquid paraffin in the space between agar, slide and cover-glass prevented drying of the agar during prolonged incubation. (This means that the bacteria were growing anaerobically most of the time; but we showed in the preceding paper, Kogut et al. 1965, that anaerobic and aerobic growth were equally affected after the previous aerobic growth in presence of dihydrostreptomycin.) Two preparations, one for control and one for dihydrostreptomycin-treated cultures, were usually placed on the same England Finder slide and incubated on the microscope stage in a hot box at $33-50^{\circ}$. With this method it was difficult to identify with certainty single bacteria, and to search the area for these in a reasonable period of time (30-60 min.) because of the few organisms originally spread over a comparatively large area, the thickness of the agar, and dust and debris on its surface which could not be avoided. We therefore started observations after a sufficient incubation period to allow for one to two divisions, and scored only units of two or more bacteria. By using phase contrast with a $\times 20$ objective and a $\times 8$ ocular throughout, we recorded the position, by letter and number of the England Finder grid, of 30-60 units of two or more bacteria, at the first search, and then relocated them after periods of incubation and scored their 'growth'. A more rapid method of scanning the slide for groups of fifty or more organisms was suggested by $\mathrm{Mr} \mathbf{M}$. Young. This consisted in viewing with a $\times 20$ objective but 95 phase, which gives a picture of lower magnification than ordinary phase and needs less precise focusing. The agar appeared as a dark background, and all debris and objects on its surface as bright spots. Among these, groups of bacteria were easily distinguished by a bluish fluorescent appearance, as well as by their shape. 


\section{RESULTS}

In the preceding paper (Kogut et al. 1965) it was reported that when aerobic cultures of Escherichia coli в were taken during exponential growth in the presence of dihydrostreptomycin but before growth had ceased, and treated to remove extracellular antibiotic, they continued to grow, aerobically as well as anaerobically, at apparently exponential though slower rates. It was also shown that the degree of inhibition of growth rate was a function of the duration of previous aerobic growth in presence of dihydrostreptomycin, and of the initial concentration of this antibiotic. Aerobically, this inhibited logarithmic growth appears to proceed for several

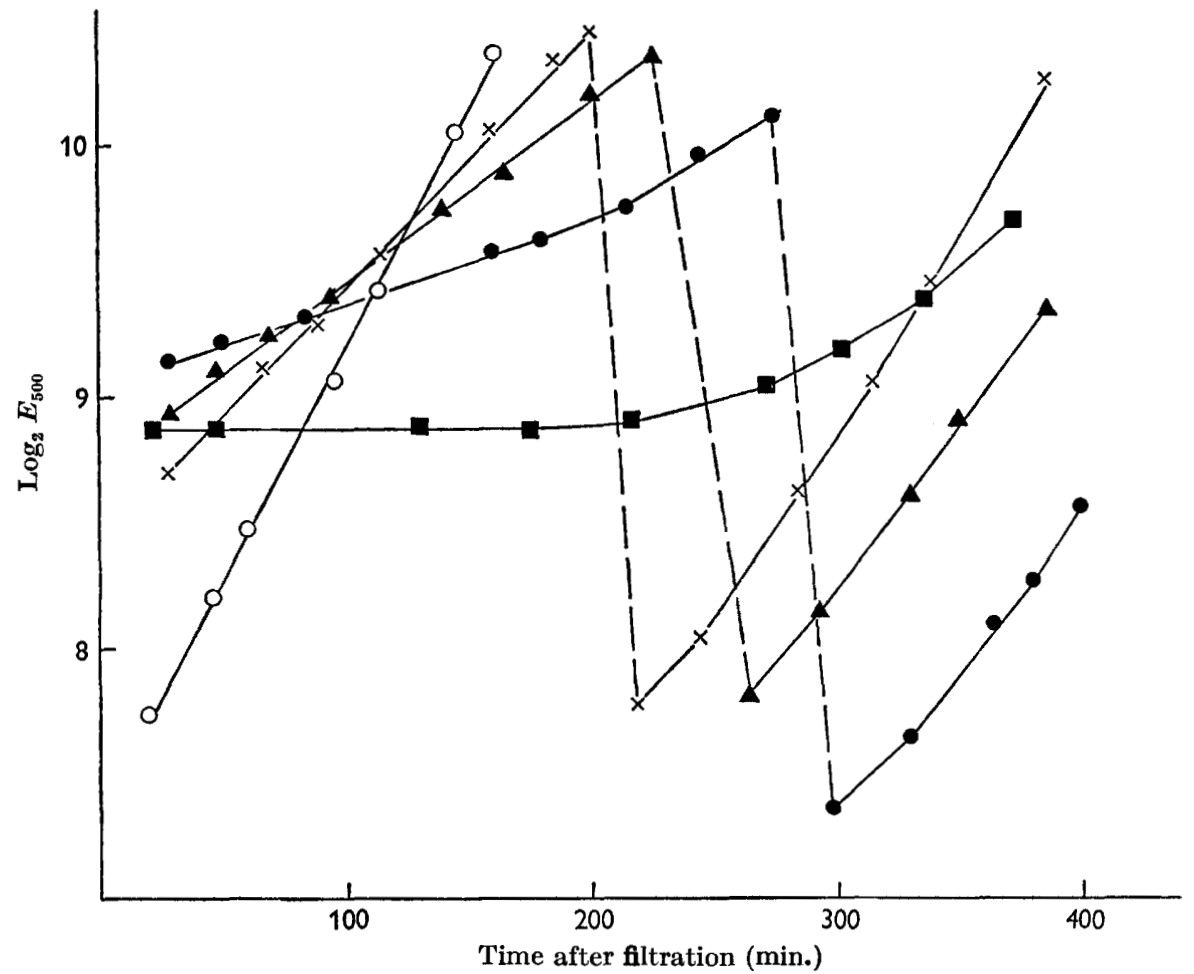

Fig. 1. Aerobic growth of Escherichia coli в after treatment with dihydrostreptomycin. Aerobic growth in antibiotic-free medium after various periods of aerobic growth in the presence of dihydrostreptomycin sulphate, $20 \mu \mathrm{g} . / \mathrm{ml}$., and subsequent removal of extracellular dihydrostreptomycin by filtration and washing. $O-O$ control culture; dihydrostreptomycin-treated cultures: $\times-\times$ filtered after 65 min.; $\Delta-\Delta$ filtered after 85 min.: - filtered after 105 min.: - -125 filtered after 125 . Dashed lines indicate dilution of cultures with fresh medium to allow for continued growth, showing recovery of growth rates.

hours, after which time the growth rates increase and gradually return to those of untreated controls. Figure 1 depicts a representative experiment of this kind, in which three of four samples continued to grow at apparently exponential inhibited rates, and all eventually recovered normal growth rates.

Two features of the inhibited growth of Escherichia coli в after treatment which 
had allowed intracellular accumulation of dihydrostreptomycin, but in the absence of extracellular antibiotic, seemed relevant to the mode of action of this antibiotic; namely, the apparently exponential nature of inhibited growth during the first few hours and the recovery in growth rates.
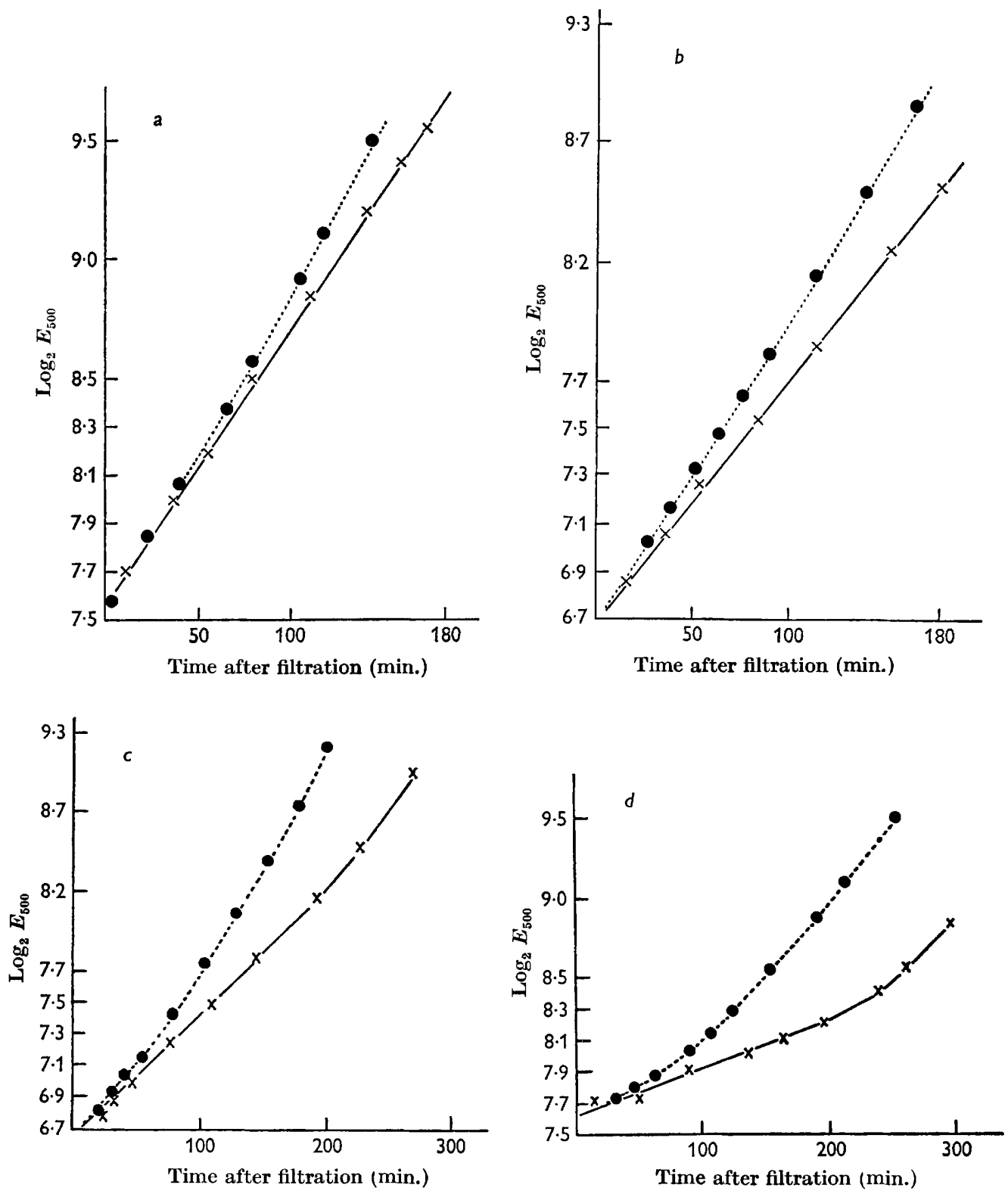

Fig. 2. Observed and calculated growth curves of Escherichia coli B after treatment with dihydrostreptomycin sulphate, $20 \mu \mathrm{g} / \mathrm{ml}$. Calculated growth curves are based on the assumption that \% inhibition of growth rate observed equals \% of population not growing and the rest growing at uninhibited rates: $a=21 \%$ inhibition of growth rate; $b=34 \%$ inhibition of growth rate; $c=\mathbf{5 2} \%$ inhibition of growth rate; $d=80 \%$ inhibition of growth rate. $x-x=$ observed growth curves; $-0=$ calculated growth curves. 
The apparently exponential nature of inhibited growth. Inspection of the records from many experiments of the above kind showed that in most cases the initial phase of inhibited growth appeared to be truly logarithmic, as far as could be determined over the periods concerned and by the methods available. We considered the

Table 1. Direct microscopic observation of multiplication of Escherichia coli $\mathrm{B}$ during periods of exponential inhibited growth and of controls (Method A)

\begin{tabular}{|c|c|c|c|c|c|}
\hline \multirow{2}{*}{\multicolumn{3}{|c|}{ Liquid culture }} & \multicolumn{3}{|c|}{ Slide culture } \\
\hline & & & & Coloulated & No of \\
\hline $\begin{array}{c}\text { Expt. } \\
\text { no. }\end{array}$ & $\begin{array}{c}\text { Growth } \\
\text { rate } \\
(\mu)\end{array}$ & $\begin{array}{c}\% \\
\text { inhibition } \\
(\mu)\end{array}$ & $\begin{array}{c}\text { Initial } \\
\text { no. of } \\
\text { organisms }\end{array}$ & $\begin{array}{c}\text { generations } \\
\text { on slide } \\
\text { (no.) }\end{array}$ & $\begin{array}{l}\text { bacteria } \\
\text { not } \\
\text { dividing }\end{array}$ \\
\hline \multicolumn{6}{|c|}{ Controls } \\
\hline $\mathbf{1}$ & $0 \cdot 80$ & 0 & 80 & $1 \cdot 6$ & 4 \\
\hline 2 & 1.00 & $\mathbf{0}$ & 18 & $3 \cdot 0$ & 1 \\
\hline $\mathbf{3}$ & 0.93 & $\mathbf{0}$ & 55 & $\mathbf{3} \cdot \mathbf{2 6}$ & $\mathbf{0}$ \\
\hline 4 & 1.05 & $\mathbf{0}$ & 65 & $2 \cdot 10$ & 3 \\
\hline
\end{tabular}

After treatment with dihydrostreptomycin sulphate, $20 \mu \mathrm{g} . / \mathrm{ml}$. for $70-95 \mathrm{~min}$.

\begin{tabular}{rrrrrr}
\hline 5 & $0 \cdot 44$ & 55 & 40 & $3 \cdot 0$ & 2 \\
6 & $0 \cdot 41$ & 58 & 38 & $3 \cdot 0$ & 2 \\
7 & $0 \cdot 33$ & 66 & 30 & $3 \cdot 3$ & 4 \\
8 & $0 \cdot 58$ & 44 & 40 & $3 \cdot 5$ & 3 \\
9 & 0.33 & 66 & 24 & $3 \cdot 0$ & 1 \\
10 & $0 \cdot 49$ & 52 & 37 & $3 \cdot 0$ & 1
\end{tabular}

Table 2. Proportions of colony-forming units/ml. in logarithmically growing cultures of Escherichia coli в

\begin{tabular}{|c|c|c|c|c|c|}
\hline $\begin{array}{c}\text { Expt. } \\
\text { no. }\end{array}$ & $\begin{array}{l}\text { Time } \\
\text { after } \\
\text { start of } \\
\text { expt. } \\
\text { (min.) }\end{array}$ & $\begin{array}{c}\text { Growth } \\
\text { rate } \\
(\mu)\end{array}$ & $E_{500}$ & $\begin{array}{r}\text { Colony- } \\
\text { forming } \\
\text { units } / \mathrm{ml} \text {. } \\
\left(\times \mathbf{1 0}^{-9}\right)\end{array}$ & $\begin{array}{c}\text { Ratio: } \\
\text { colony- } \\
\text { forming } \\
\text { units } / \mathrm{ml} . / \\
\boldsymbol{E}_{500} \\
\left(\times 10^{-9}\right)\end{array}$ \\
\hline 1 & 35 & 0.98 & 0.311 & 0.611 & 1.96 \\
\hline 2 & 135 & $1 \cdot 0$ & 0.44 & 0.86 & 1.92 \\
\hline $\mathbf{3}$ & 60 & 0.96 & 0.485 & $0 \cdot 85$ & $1 \cdot 75$ \\
\hline 4 & 60 & $1 \cdot 00$ & 0.383 & 0.733 & $1 \cdot 83$ \\
\hline 5 & 60 & $1 \cdot 00$ & 0.293 & 0.58 & $\mathbf{1 . 9 7}$ \\
\hline 6 & 75 & $1 \cdot 03$ & 0.320 & 0.54 & $I \cdot 69$ \\
\hline 7 & 75 & $1 \cdot 06$ & 0.345 & 0.766 & $2 \cdot 22$ \\
\hline \multirow[t]{2}{*}{8} & 0 & 1.07 & $0 \cdot 150$ & 0.305 & $2 \cdot 01$ \\
\hline & 150 & 1.07 & 0.966 & $1 \cdot 82$ & $1 \cdot 89$ \\
\hline \multirow[t]{2}{*}{9} & 0 & 1.08 & $0 \cdot 156$ & $0 \cdot 267$ & 1.75 \\
\hline & 90 & 1.08 & 0.597 & $1 \cdot 06$ & $1 \cdot 77$ \\
\hline
\end{tabular}

Mean value for colony-forming units $/ \mathrm{ml} . / E_{500}=1.98 \times 10^{9}$.

possibility that such apparently exponential growth curves might be obtained if a proportion of the population were not growing at all, and the rest were growing at uninhibited rates. Figure 2 compares growth curves calculated on the assumption that $x \%$ of the bacteria were not growing at all and the remainder were growing 
at uninhibited rates, with those curves actually observed in four experiments, where growth rates were inhibited by $x \%$. It is clear in Fig. 2 that for inhibition of growth rates by more than $20 \%$, the two sets of growth curves are easily distinguishable. Figure 2 also shows that for each observed growth curve, if $x \%$ of the bacteria in the population had not been growing at all, then the rest could not have been growing at uninhibited rates; and if the \% of non-growing bacteria was smaller

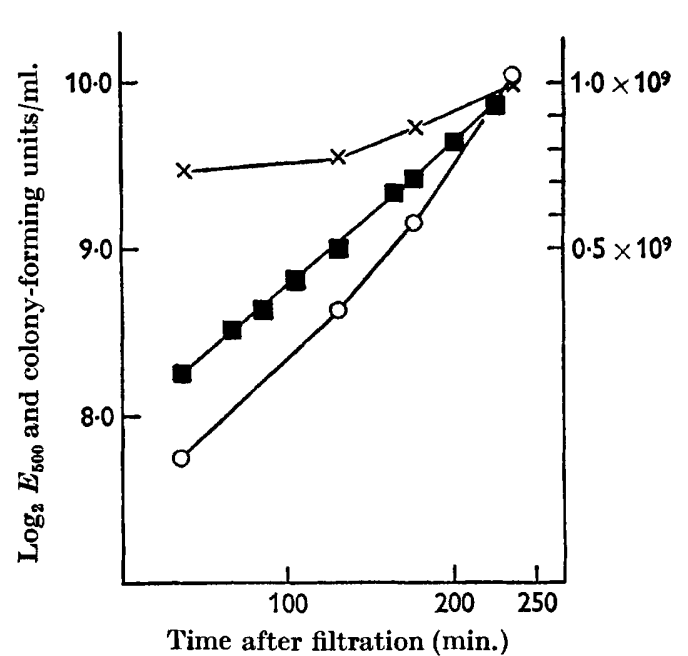

Fig. 3

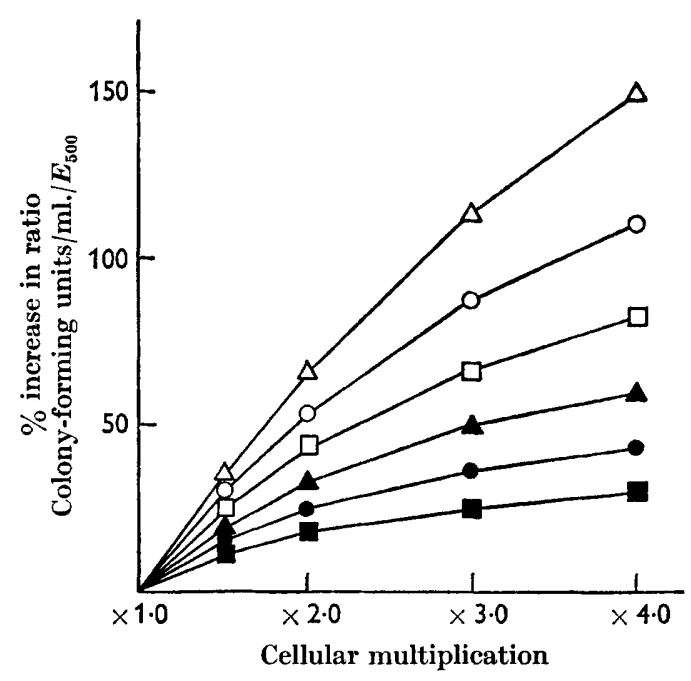

Fig. 4

Fig. 3. Relation between $E_{500}$ and colony counts after treatment of Escherichia coli B with dihydrostreptomycin. Effect of aerobic growth for $75 \mathrm{~min}$. in the presence of dihydrostreptomycin, $20 \mu \mathrm{g} / \mathrm{ml}$., on subsequent increase in extinction, colony-forming units $/ \mathrm{ml}$. and ratio of colony-forming units $/ \mathrm{ml}$. to $E_{500}$, after removal of extracellular dihydrostreptomycin. $\square=\log _{2} E_{500} ; 0-\mathrm{O}=\log _{2}$ colony-forming units $/ \mathrm{ml}$. (lefthand scale). $\times-\times=$ colony-forming units $/ \mathrm{ml}$. $/ E_{500}$ (right-hand scale).

Fig. 4. Relation between colony counts and extinction during growth of populations containing non-dividing bacteria. Percentage increase in the ratio of colony-forming units $/ \mathrm{ml}$. to $E_{500}$ as a function of cellular multiplication, calculated for populations assumed to contain various proportions of bacteria which are not multiplying. Proportions of populations assumed to be not growing $(\%)$ : $\square-\square=30 ; 0-0=40$; $\Delta-\Delta=50 ; \square-\square=60 ; 0-0=70 ; \triangle-\triangle=80$.

than $x \%$, the growth rates of the remainder would have to be even slower. In other words, for any population of bacteria containing only those growing normally or not at all, to yield growth curves of the kind observed, the percentage of 'dead' bacteria would have to be larger than the \% inhibition of growth rates.

Whether such a situation existed could be tested directly by observing the fate of individual bacteria during periods corresponding to the phase of apparently exponential, but slower growth in liquid culture. With the method $\mathbf{A}$, outlined in Methods, the number of bacteria which had not divided at all, could be evaluated. Table 1 shows that the numbers of bacteria (out of those originally placed on the slides) which had not divided at all in about three generation times were similar in the dihydrostreptomycin-treated cultures and in controls.

Examination of the proportion of bacteria able to give visible colonies. A further 
test for the possibility that a certain percentage of the population were not multiplying at all, would be provided by examination of the proportion of bacteria able to give visible colonies. In untreated, exponentially-growing cultures of Escherichia coli the colony count increased in parallel with other measurements of growth,

Table 3. Relation of colony counts and extinction measurements in growing cultures of Escherichia coli в after treatment with dihydrostreptomycin sulphate, $20 \mu \mathrm{g} . / \mathrm{ml}$

Observed changes in ratio of colony-forming units $/ \mathrm{ml} . / E_{500}$ with time after treatment with dihydrostreptomycin, compared with calculated changes. Calculations are based on the assumption that $\%$ decrease in ratio of colony-forming units $/ \mathrm{ml} . / \boldsymbol{E}_{500}$ from controls $=$ $\%$ of population not multiplying, and the changes in ratio of colony-forming units $/ \mathrm{ml}$./ $E_{500}$ during growth shown in Fig. 4.

Expt. no. … … … …

Growth in dihydrostreptomycin (min.)

Growth rates $(\mu)$ after filtration

Inhibition of growth rates compared to controls (\%)

Observed changes during growth after filtration

Sample 1

T1 time after filtration (min.)

E1 $E_{500}$

C1 colony-forming units $/ \mathrm{ml} .\left(\times 10^{-9}\right)$

35

$0 \cdot 308$

$0 \cdot 229$

35

$0 \cdot 308$

$0 \cdot 229$

0.705

60

$0 \cdot 401$

$0 \cdot 465$

$1 \cdot 160$

0

0.381

$0 \cdot 144$

$0 \cdot 378$

Sample 2

T2 time after $\mathbf{T} 1$ (min.)

E2 $E_{500}$

95

C2 colony-forming units $/ \mathrm{ml}$. $\left(\times 10^{-9}\right)$

95

140

$\mathbf{0} \cdot 400$

$0 \cdot 682$

75

0.570

115

CE 2 colony-forming units $/ \mathrm{ml} . / \mathrm{E}_{500}\left(\times 10^{-9}\right)$

$0 \cdot 770$

$0 \cdot 580$

$0 \cdot 730$

0.471

$\mathbf{0} \cdot \mathbf{8 5 0} \quad \mathbf{1} \cdot \mathbf{2 8 0}$

$0 \cdot 168$

$0 \cdot 356$

Calculations
(a) $\frac{\mathrm{CE} 0^{*}-\mathrm{CE} 1}{\mathrm{CE} 0} \times 100=\begin{gathered}\% \text { decrease in CE } 1 \\ \text { from controls }\end{gathered}$
(b) $\mathrm{C2} / \mathrm{Cl}=$ cellular multiplications (c) $\frac{\mathrm{CE2}-\mathrm{CE} 1}{\mathrm{CE} 1} \times 100=\%$ change in ratio units $/ \mathrm{ml}$. $/ \boldsymbol{E}_{\mathbf{5 0 0}}$ between

$\begin{array}{cccc}62.6 & 62.6 & 38.6 & 80.0 \\ 1.748 & 2.540 & 1.570 & 1.167 \\ +9.2 & +20.6 & +10.3 & -6.2\end{array}$
$\mathrm{S} 1$ and $\mathrm{S} 2$

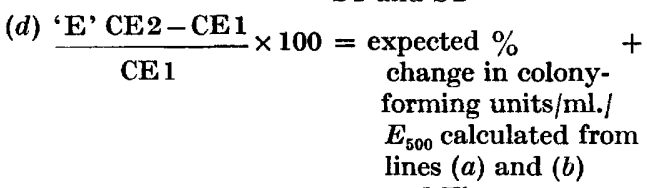 and Fig. 4
(e) ' $\mathrm{E}$ ' $\mathrm{CE} 2$ = expected ratio of colony- observed CE 1 and line $(d)\left(\times 10^{-9}\right)$
* $\mathrm{CE} 0=$ colony-forming units $/ \mathrm{ml}$. $/ E_{50 \mathrm{u}}$ for controls, from Table $2=1.9 \times 10^{9}$.

e.g. extinction $\left(E_{500}\right)$ and the ratio of colony count to $E_{500}$, which is thus a measurement of the proportion of bacteria in the culture able to give visible colonies, was found to be constant (see Table 2). If, after treatment with dihydrostreptomycin, and removal of extracellular antibiotic, some bacteria were unable to multiply at all, and others were multiplying at normal, or even at inhibited rates, then the ratios of colony counts (colony-forming units $/ \mathrm{ml}$.) $/ E_{500}$ of such cultures should be 
increasing with time, at rates determined by the proportions of non-multiplying bacteria and the growth rates of the remainder. Figure 3 shows the rate of change of $E_{500}$ and colony-forming units/ml. (both plotted as $\log _{2}$ ) and of the ratio of colonyforming units $/ \mathrm{ml} . / E_{500}$ of a culture filtered after aerobic growth for $75 \mathrm{~min}$. in the presence of $20 \mu \mathrm{g}$. dihydrostreptomycin sulphate $/ \mathrm{ml}$. when the apparently logarithmic growth rate was inhibited by approximately $50 \%$. Although the ratio of colonyforming units/ml./ $E_{500}$ was much lower than the mean control value (by about $60 \%$ ) it remained more or less constant during most of the phase of logarithmic but inhibited growth.

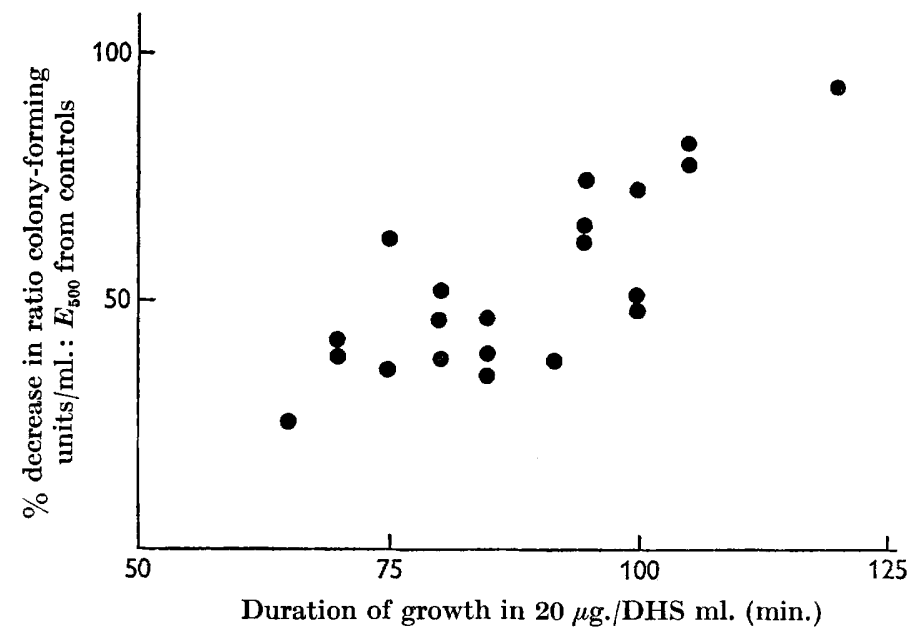

Fig. 5. Loss of colony-forming ability of Escherichia coli $\mathrm{B}$ as a function of dihydrostreptomycin(DHS) treatment. Percentage decrease in ratio of colony-forming units/ml. to $E_{500}$ from control values, plotted against duration of previous growth in the presence of dihydrostreptomycin sulphate, $20 \mu \mathrm{g} . / \mathrm{ml}$.

It can be calculated that if $x \%$ of the bacteria in a population were not multiplying, then the ratio of colony-forming units $/ \mathrm{ml} . / \boldsymbol{E}_{500}$ should increase during growth as illustrated in Fig. 4. With the use of this graph, and assuming that \% decrease in colony-forming units $/ \mathrm{ml} . / E_{500}$ from controls $=\%$ of population not multiplying, one can calculate the expected increase in colony-forming units $/ \mathrm{ml} / \boldsymbol{E}_{500}$ for any interval of growth, from the experimental data on growth rates and colony-forming units $/ \mathrm{ml} . / \boldsymbol{E}_{500}$. Table 3 compares these calculated changes in the ratio of colonyforming units $/ \mathrm{ml} . / E_{500}$ with those actually recorded in a number of experiments. It is clear that the majority of bacteria in these populations must have been multiplying throughout the periods of 'logarithmic but inhibited growth' when the samples were taken. However, when the extent of decrease in colony-forming units $/ \mathrm{ml} . / E_{500}$ (as $\%$ of control values) after treatment with dihydrostreptomycin was determined in many experiments, it was found to be a function of the duration of previous aerobic growth in the presence of the antibiotic (as shown in Fig. 5) in the same way as was \% inhibition of growth rates.

There was thus an apparent paradox in the behaviour of such dihydrostreptomycin-treated cultures. On the one hand growth of liquid cultures (measured by $E_{500}$ ) direct microscopic observation of individual organisms for three generation 
times, and the constancy of the ratio colony-forming units $/ \mathrm{ml} . / E_{500}$ during this period, suggested that nearly all the bacteria in such cultures were initially able to multiply. On the other hand experiments which measured the proportion of bacteria able to give visible colonies after prolonged incubation on solid media showed these to be reduced in such dihydrostreptomycin-treated cultures.

These discrepancies might have arisen from differences in the methods involved. Thus some of the bacteria in the antibiotic-treated cultures (their number varying with the duration of treatment) might have been damaged in such a way that some factor involved in the colony count technique, but not in the slide or liquid culture, rendered them unable to divide. Such a situation was described for phenol-treated Escherichia coli, including strain B, by Harris \& Whitefield (1963). Two such possible factors, namely, altered temperature sensitivity and adherence to glassware, as a result of dihydrostreptomycin-treatment, were considered by us. By using the Miles \& Misra drop technique, to avoid contact of bacteria with molten agar, portions of the final dilutions for plating were incubated, for $10 \mathrm{~min}$. each, at room temperature $\left(18^{\circ}\right), 40,45,48$ and $50^{\circ}$. At incubation temperatures of $48^{\circ}$ and $50^{\circ}$, all cultures showed a decrease in colony count, but in the dihydrostreptomycintreated cultures this was more marked than in the controls. After incubation at $40^{\circ}$ and $45^{\circ}$, controls and dihydrostreptomycin-treated bacteria behaved similarly; all showed small and variable decreases in colony counts as compared to those incubated at room temperature, but this did not affect significantly the loss in proportion of colony-forming units $/ \mathrm{ml} . / E_{500}$ following antibiotic treatment. In general, and with $10 \mathrm{~min}$. of incubation at room temperature, the loss in proportion of colony-forming units after dihydrostreptomycin treatment appeared slightly larger with the Miles \& Misra drop technique than in pour plates (of the order of 10-15\%). In the pour-plate method, the agar was kept in a water bath at $41^{\circ}$ before pouring.

If increased adherence to glass were to account for the apparent reduction in viable units, then the percentage loss would increase with the number of serial dilutions used. In the Miles \& Misra drop technique the aim is to count about 30 colonies from drops of $0.02 \mathrm{ml}$, whilst in the plate counts dilutions to about 100 colony-forming units $/ \mathrm{ml}$. are used. The latter method, therefore, involves a larger number of serial dilutions, and should give the greater \% decrease in colonyforming units $/ \mathrm{ml} . / \boldsymbol{E}_{500}$. This was not the case.

The possibility that an increased tendency to clump could account for the apparent decrease in colony-forming units $/ \mathrm{ml} / \boldsymbol{E}_{500}$ with dihydrostreptomycintreated bacteria, was considered to be unlikely; only occasionally slight clumping was observed in direct microscopic examination and incorporation of 'Lissapol' (Norris $\&$ Powell, 1961) into the diluents did not affect the results of plate counts.

A different interpretation of the discrepancy between logarithmic growth in liquid cultures at inhibited rates, coupled with the ability of all bacteria in the cultures to divide for a time, and the inability of a proportion of these bacteria to give visible colonies in conventional plate counts, may be suggested. This is that at some time, corresponding to the end of the period of inhibited logarithmic growth, a proportion (varying in size with the duration of antibiotic treatment) of the bacteria in such populations ceases to divide.

Direct microscopic examination of individual bacteria during prolonged incubation 
on nutrient agar. Method B (see Methods) allowed continuous or intermittent observation of several clones over many generations, from the first or second division to the stage of visible colony formation. (With this method $\mathrm{B}$ we did not score single bacteria because these could not be identified with sufficient certainty, but started observations on bacteria which had undergone at least one division.) It was readily established with this technique that the decrease in colony-forming units $/ \mathrm{ml} . / E_{500}$ in the dihydrostreptomycin-treated cultures was not due to arrest of growth at a late, micro-colony stage: once a group of 8-10 bacteria had been located

Table 4. Division and growth of bacteria from dihydrostreptomycin-treated cultures of Escherichia coli $\mathrm{в}$ during inhibited logarithmic growth and from control cultures, observed microscopically on solid medium (Method B)

\begin{tabular}{|c|c|c|c|c|c|c|}
\hline Expt. no. ... & \multicolumn{2}{|l|}{1} & \multicolumn{2}{|c|}{$\mathbf{2}$} & \multicolumn{2}{|c|}{3} \\
\hline $\begin{array}{l}\text { Growth in dihydrostreptomycin sulphate, } \\
20 \mu \mathrm{g} . / \mathrm{ml} \text {. (min.) }\end{array}$ & \multicolumn{2}{|l|}{90} & \multicolumn{2}{|l|}{60} & \multicolumn{2}{|l|}{65} \\
\hline Growth rates $(\mu)$ & \multicolumn{2}{|c|}{$0 \cdot 110$} & \multicolumn{2}{|c|}{0.540} & \multicolumn{2}{|c|}{$0 \cdot 434$} \\
\hline$\%$ inhibition $(\mu)$ & \multicolumn{2}{|l|}{89} & \multicolumn{2}{|l|}{49} & \multicolumn{2}{|c|}{$53 \cdot 6$} \\
\hline $\begin{array}{l}\% \text { decrease in ratio colony-forming } \\
\text { units } / \mathrm{ml} . / E_{500} \text { from control }\end{array}$ & \multicolumn{2}{|l|}{$86 \cdot 8$} & \multicolumn{2}{|c|}{$31 \cdot 4$} & \multicolumn{2}{|c|}{$36 \cdot 0$} \\
\hline Time of sampling after filtration (min.) & \multicolumn{2}{|c|}{90} & \multirow{2}{*}{\multicolumn{2}{|c|}{$\begin{array}{l}70 \\
32-35^{\circ}\end{array}$}} & \multicolumn{2}{|c|}{70} \\
\hline \multirow[t]{2}{*}{ Incubation temperature of slide } & $33-3$ & $35^{\circ}$ & & & 30 & $-35^{\circ}$ \\
\hline & Control & $\begin{array}{l}\text { DHS- } \\
\text { treated }\end{array}$ & Control & $\begin{array}{l}\text { DHS- } \\
\text { treated }\end{array}$ & Control & $\begin{array}{l}\text { DHS- } \\
\text { treated }\end{array}$ \\
\hline $\begin{array}{l}\text { Calculated generations on slide at first } \\
\text { observations }\end{array}$ & $2+$ & $0 \cdot 5$ & $2-2 \cdot 75$ & $2 \cdot 4$ & $\mathbf{1} \cdot \mathbf{5}-\mathbf{3} \cdot \mathbf{5}$ & $1 \cdot 4-3 \cdot 4$ \\
\hline $\begin{array}{l}\text { Growth stage (bacteria/group) at first } \\
\text { observations }\end{array}$ & $4-20$ & $1-8$ & $2-8$ & 2-20 & 2-8 & $2-20$ \\
\hline $\begin{array}{l}\text { Nos. first judged 'positive' as bacteria } \\
\text { having divided }\end{array}$ & 54 & 26 & 29 & 40 & $\mathbf{2 8}$ & 17 \\
\hline Nos. first judged 'doubtful' & 8 & 27 & 2 & 7 & 4 & $\mathbf{5}$ \\
\hline Nos. later confirmed as colonies & 62 & 16 & 28 & 34 & 27 & 11 \\
\hline Nos. later judged 'not grown' & $\mathbf{1}$ & 29 & 2 & 11 & 4 & 11 \\
\hline Nos. lost & $\mathbf{0}$ & 8 & $\mathbf{1}$ & 2 & 1 & $\mathbf{0}$ \\
\hline $\begin{array}{l}\% \text { of those first recorded, later not } \\
\text { grown }\end{array}$ & $\mathbf{0}$ & 70 & 10 & $27 \cdot 5$ & 16 & 50 \\
\hline$\%$ of those first judged 'positive' later & $\mathbf{0}$ & $38 \cdot 5$ & $3 \cdot 5$ & 15 & 3 & 35 \\
\hline
\end{tabular}

during the first few hours of incubation on the slide, it always continued to grow and divide; occasionally some (10-20\%) of the clones in the treated cultures appeared somewhat retarded, but they eventually reached the same colony size (diameter) as did those of controls. This left us with the question about what happens during the first few generation times after the removal of extracellular antibiotic. We have shown that with method A one cannot distinguish the dihydrostreptomycin-treated from control cultures on the basis of \% of bacteria which undergo no division during three generation times. With method B, however, there was a difference between control and treated cultures. In the former, bacteria which had divided at least once could be recognized with almost complete certainty, and practically all the organisms scored initially as two or more bacteria continued to grow and divide. In the dihydrostreptomycin-treated cultures it was much more difficult to recognize such small groups of bacteria; a much larger proportion of those recorded initially 
(particularly of those considered 'doubtful') later could not be recognized or remained unchanged. Table 4 shows a number of such observations on control and dihydrostreptomycin-treated cultures. Recorded are the approximate number of generation times of incubation on the slide at the first observation (calculated from the growth rates in liquid culture) and the growth stage actually reached at the first observation (in numbers of bacteria per group). The wide spread in the latter values can be partly accounted for by the length of time it took to search the slide at this stage (1-3 hr) and partly by the distribution of division times and growth rates within populations. The groups of organisms first recorded are subdivided into those 'judged positively as bacteria having divided' and those classified as 'doubtful' (these latter were usually the smaller groups of bacteria, as mentioned above). In dihydrostreptomycin-treated cultures, none of those first judged as 'doubtful' were later found to have grown, whilst in the controls some of these did later give colonies (expt. 1). Of those first judged as 'positive' in the control cultures, almost all grew to colonies, whereas in the treated cultures a proportion was later found to be of doubtful appearance, unchanged in size, or could no longer be recognized. These observations indicate that in the dihydrostreptomycin-treated cultures, a proportion of bacteria did not divide more than once or twice and that such bacteria may change in appearance, or in some cases lyse, so that not all of these are recorded. Postgate et al. (1961) reported that populations of Aerobacter aerogenes of intermediate viability (20-70\%) contained a proportion of organisms which appeared to attempt multiplication but failed, and were seen as 'drum-stick or spherical bodies of low contrast and not easily recognized'. Because of this suggestion of altered appearance, the values for 'proportion of bacteria which stopped growing' in Table 4, must be minimal, since one cannot estimate the number of those which might have ceased to divide and changed their appearance by the time the first observations were made.

\section{The recovery in growth rates after inhibited exponential growth of Escherichia coli $\mathrm{B}$ in the absence of extracellular antibiotic}

This second feature of growth of dihydrostreptomycin-treated cultures of Escherichia coli $\mathrm{B}$ in the absence of extracellular antibiotic, the recovery of growth rates, is illustrated in Fig. 1 and occurred in all cases except where growth was completely inhibited. To observe the whole of this recovery phase and to determine whether the growth rates actually returned to normal, cultures had to be diluted with fresh medium at times when it was judged from extinction measurements that they were nearing exhaustion of nutrients. Under these conditions they returned to normal or very nearly normal growth rates.

Such growth curves showing apparent recovery, at first suggested outgrowth of streptomycin-resistant, or of uninhibited organisms present in the culture at the time of filtration. Duplicate platings for colony counts on the ordinary agar medium and on agar medium + dihydrostreptomycin sulphate $20 \mu \mathrm{g} . / \mathrm{ml}$. were made, on controls and at various times after addition of dihydrostreptomycin. Table 5 shows that in control cultures such resistant organisms numbered about $1-3 / 10^{8}$ colonyforming units. In the treated cultures, the highest proportion of resistants found was $20 / 10^{8}$ colony-forming units, but there was no consistent accumulation of resistant organisms over total viable organisms with duration of growth (difference 
Table 5. Occurrence and growth of dihydrostreptomycin-resistant bacteria in controls and dihydrostreptomycin-treated cultures of Escherichia coli $\mathbf{B}$

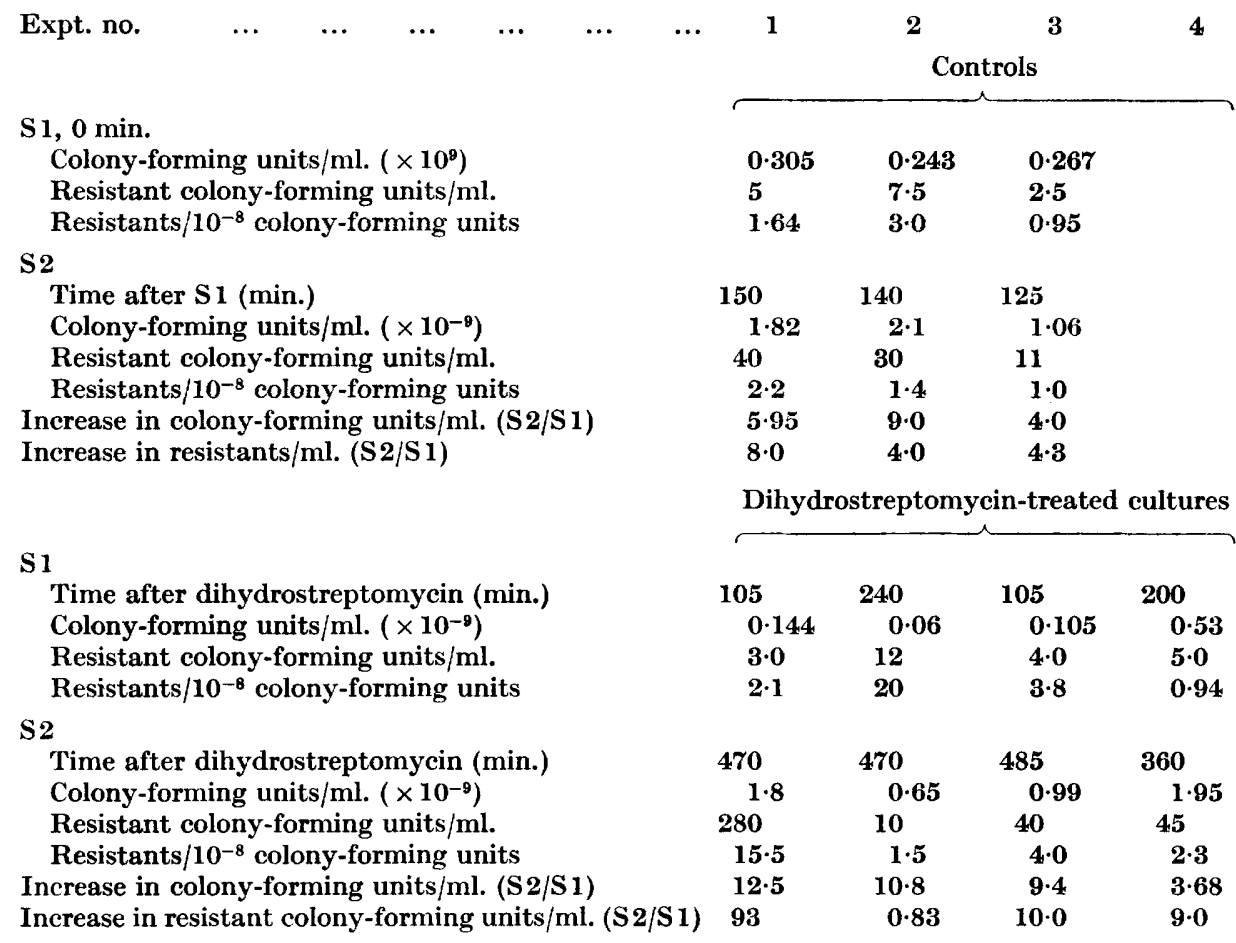

Table 6. Relation between colony counts in recovery phase and during period of inhibited expontential growth

Calculation of $\%$ of colony-forming units present during inhibited exponential growth phase which could account for colony-forming units present in recovery phase, had they multiplied at uninhibited rates throughout (see text).

Expt. no.

S 1

Time after dihydrostreptomycin (min.)

$E_{500}$

Colony-forming units/ml. $\left(\times 10^{-9}\right)$

S2

Time after dihydrostreptomycin (min.)

$E_{500}$

Colony-forming units/ml. $\left(\times 10^{-9}\right)$

S2-S1

Time (hr)

Generations observed

Generations calculated if $\mu=1 \cdot 0$

Calculated colony-forming units $/ \mathrm{ml}$. at $\mathrm{S} 1$, if all

those counted at $\mathrm{S} 2$ had arisen at $\mu=1 \cdot 0\left(\times 10^{-9}\right)$

As $\%$ of observed colony-forming units/ml. at S1

1

2

3

4

105

$0 \cdot 381$

105

$0 \cdot 144$

$0 \cdot 310$

200

$0 \cdot 465$

140

1

0.53

470

1.286

$\begin{array}{ll}1.8 & 0.99\end{array}$

360

$1 \cdot 282$

310

1.95

$1 \cdot 018$

$6 \cdot 08$

$6 \cdot 33$

$2 \cdot 66$

$1 \cdot 43$

\section{$3 \cdot 64$}

$3 \cdot 24$

$1 \cdot 88$

$2 \cdot 83$

$6 \cdot 08$

$6 \cdot 33$

$\mathbf{2 \cdot 6 6}$

$1 \cdot 62$

$0 \cdot 027$

$0 \cdot 012$

0.308

$\mathbf{2} \cdot 83$ 
between $S 1$ and S2). Only in one case (expt. 1) was there a greater increase in the number of resistant organisms/ml. (93-fold) than in the total colony count $/ \mathrm{ml}$. (12.5-fold) during $400 \mathrm{~min}$. of growth. In this experiment, the last count, at $470 \mathrm{~min}$. after adding dihydrostreptomycin, was well within the recovery phase of the growth curve, but it can be easily seen that 280 resistant organisms $/ \mathrm{ml}$. would have been quite undetectable by extinction measurements $\left(E_{500} 1 \cdot 0=1.9 \times 10^{9}\right.$ colony-forming units $/ \mathrm{ml}$.). It is obvious, therefore, that the apparent recovery in growth rates
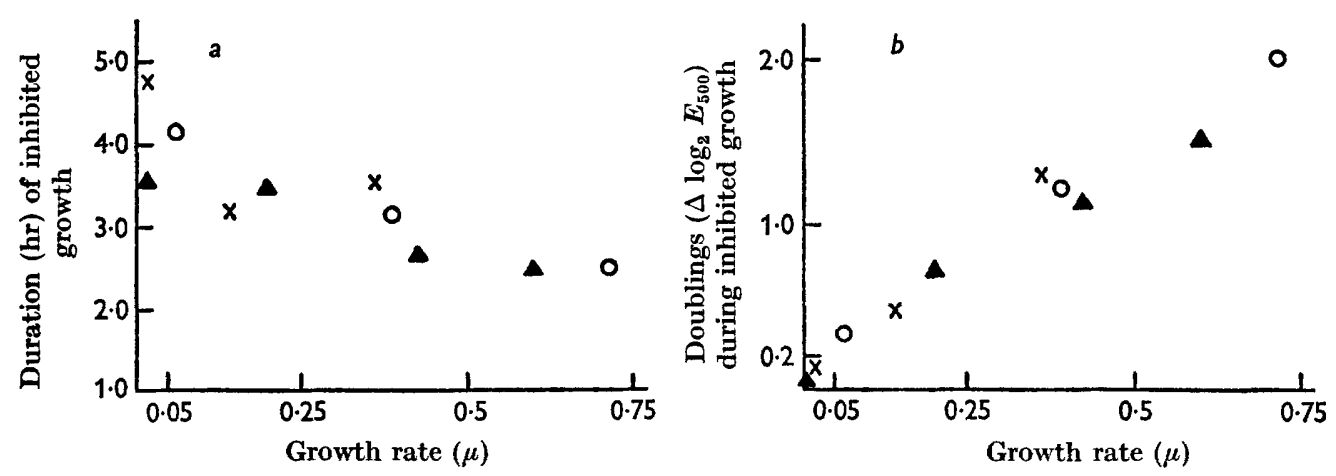

Fig. 6. Duration and extent of inhibited exponential growth of Escherichia coli B before onset of recovery as a function of growth rates. (a) Duration of inhibited exponential growth before onset of recovery. (b) Doublings, measured as $\Delta \log _{2} E_{500}$, before onset of recovery. Different symbols are from different experiments.

which usually followed inhibited logarithmic growth after treatment with dihydrostreptomycin and removal of extracellular antibiotic, could not have been due to outgrowth of pre-existing organisms resistant to dihydrostreptomycin $20 \mu \mathrm{g} . / \mathrm{ml}$. or to sudden bursts of mutations giving resistant organisms in sufficient numbers.

Regarding the possibility that continued uninhibited growth of some bacteria might account for the observed recovery phase, it has already been shown by consideration of Fig. 2 that if the observed growth curves were contributed in part by growth of individual organisms at uninhibited rates, then the proportions of nonmultiplying bacteria would have to be larger than those found. It can also be calculated from experiments in which colony-forming units $/ \mathrm{ml}$. were determined in the recovery phase as well as during the phase of inhibited logarithmic growth (Table 6) that if all those colony-forming units counted in the recovery phase had arisen at uninhibited rates $(\mu=1)$ then they could have constituted only a fraction of the bacteria counted during the logarithmic phase of inhibited growth as able to give visible colonies.

It would appear therefore, that the observed increases in growth rates, whether measured by extinction or colony-forming units (Fig. 3) must represent genuine recovery of at least a substantial portion of the treated populations. It would seem reasonable to assume that such recovery from inhibition would set in after a certain amount of cell synthesis had occurred; in other words that it would depend on the number of cell-multiplications, so that the inhibited, or inhibitory, factors were diluted out by multiplication. In such a case one would expect the onset of recovery to appear after a given number of doublings in bacterial mass or numbers, irrespec- 
tive of the growth rates, and hence to occur later on the time scale in the more inhibited cultures. Although the time of onset of recovery cannot be evaluated with precision, inspection of Fig. 1 shows that it was more or less the same in all the samples, whilst total cell synthesis (difference in $\log _{2} E_{500}$ between filtration and onset of recovery) which had occurred at this time varied in the different samples. In Fig. 6 the duration of inhibited logarithmic growth before onset of recovery (Fig. $6 a$ ) and the total cell-synthesis during this period (expressed as doublings) (Fig. $6 b$ ) are plotted against the growth rates for several experiments. It can be seen in Fig. $6 a$ that for a 20 -fold change in growth rate, the estimated time of onset of recovery varied only about 2 -fold. The doublings in cell mass, synthesized during inhibited growth, and before the onset of recovery, however, varied directly with the growth rate. Such a situation suggests that recovery must be a consequence of some process which is not inhibited, during the time that exponential growth is inhibited, by the treatment with dihydrostreptomycin.

\section{DISCUSSION}

Most of the literature on the mode of action of streptomycin deals with experiments where the uptake of the antibiotic by the cells is continuous, so that intracellular concentrations are not constant but increasing; hence the inhibition of growth appears to be cumulative. Under these conditions, factors which influence the intracellular accumulation of antibiotic cannot be distinguished from those which affect its intracellular mode of action, and many of the diverse effects of environmental factors on the antibiotic action of streptomycin and dihydrostreptomycin reported in the literature, might have been due to effects on their uptake into the cells, and hence their intracellular concentrations (Kogut \& Lightbown, 1964a).

The present and earlier studies (Kogut \& Lightbown, 1963, 1964b) have shown that after treatment of Escherichia coli B with low concentrations of dihydrostreptomycin under conditions where uptake and intracellular accumulation are known to occur (Hancock, 1962 $a$; Hurwitz \& Rosano, 1962) and subsequent removal of extracellular antibiotic at various times, the cultures continue to grow at slower but exponential rates for periods of several hours, after which the growth rates gradually return to normal. Dubin \& Davis (1962) used a similar technique to study what they described as 'streptomycin-triggered' effects. They apparently assumed that filtration and washing removed the intracellular as well as the extracellular antibiotic. We, on the other hand, assume, supported by the data of Hancock (1962a), that after such filtration and washing treatments, the cultures continue to grow in the presence of more or less constant amounts of intracellular antibiotic. However, cultures are populations of individuals which vary, within certain limits, in physiological properties, so that individual values are distributed around a mean. The parameters which are usually measured, such as growth rates, are mean values, and the intracellular concentrations of dihydrostreptomycin in the individual bacteria of such cultures should also be distributed around a mean. The extent of inhibition of exponential growth in treated populations was found to be a function of the duration of previous aerobic growth in a given external concentration $(\mu \mathrm{g} . / \mathrm{ml}$.) of dihydrostreptomycin. It seems reasonable to assume that it is a function of the mean intracellular concentration of the drug in the individuals of 
the population. However, although such populations grow exponentially but at slower rates for several hours, colony count data and direct microscopic observations show that later some of the bacteria cease to multiply, whilst others multiply at accelerating rates and eventually return to normal growth rates. The proportion of bacteria in these two fractions (estimated from measurements of colony-forming units $\left./ \mathrm{ml} . / E_{500}\right)$ was almost constant during the exponential phase of inhibited growth, and was a function of the duration of previous growth in a given concentration of dihydrostreptomycin. Hurwitz, Landau \& Doppel (1962) claimed that the loss of ability to form macro-colonies after exposure of Escherichia coli to streptomycin closely paralleled the loss of ability to divide at all. They correlated the $\%$ of bacteria from streptomycin-treated cultures which did not give visible colonies in colony counts with the $\%$ of bacteria which did not undergo division when observed microscopically during incubation for $90 \mathrm{~min}$. on slides covered with nutrient agar. They treated cultures in nutrient broth with $1 \mu \mathrm{g}$. streptomycin $/ \mathrm{ml}$. for $1,17.5$ and 35 min. Since they gave no indication of how this treatment affected the growth rates of their populations, and since there were no data for untreated controls, it is difficult to evaluate whether incubation for $90 \mathrm{~min}$. on the slides was sufficient for any division to have occurred. From our experience it would seem that in their third experiment, where they state that $99 \%$ of the population was unable to give macro-colonies, the growth rate must have been similarly inhibited, so that the mean generation time would have been increased by a factor of about 10, and no division would be expected to occur during $90 \mathrm{~min}$. of incubation.

It seems to us of importance, at this point, to examine the significance and interpretation of 'viable count' measurements in relation to antibiotics. To state the obvious, the technique of 'viable', or colony counting, measures the number of organisms, or units, in a measured sample of a population which can grow and divide sufficiently to give visible colonies. When a growth-inhibitory substance, such as dihydrostreptomycin, or streptomycin is added to a population, and samples removed for viable counting, then provided that a sufficient dose were taken up rapidly by all the individuals, and this was not removed from the bacteria by the dilutions and washing involved in the technique, the viable count should immediately fall to zero. This is not usually the case, mainly because the bacteria do not accumulate growth-inhibitory concentrations of the drug instantaneously. In such cases, e.g. with such growth-inhibitory substances, 'viable counts' then measure the number of units in the population which do not contain growth-inhibitory concentrations. The use of the term 'bactericidal' when referring to the effects of antibiotics which are known to be growth-inhibitory under the experimental conditions used, seems meaningless, unless one could ensure that all, including the intracellularly held, antibiotic were removed from the organisms prior to plating; or its use must be merely operational, denoting the irreversibility of binding at the inhibitory sites. In the case of streptomycin, available evidence (Hancock, $1962 a, b$, Spotts, 1962) suggests in fact that it is very tightly bound to some inhibitory sites. The decline in 'viable count', and in our case, the reduction in the ratio of colonyforming units $/ E_{500}$, as a function of the duration of treatment with the antibiotic, must therefore reflect the rate of acquisition and retention of growth-inhibitory antibiotic concentration by individuals in the population. In other words, the ratio of colony-forming units/ml./ $E_{500}$ after a given treatment with dihydrostreptomycin 
should measure the proportions of bacteria in the treated population whose intracellular concentrations of the drug are insufficient to inhibit growth completely.

It has been suggested that streptomycin (and dihydrostreptomycin) acts by combining with ribosomes, free from messenger $\mathrm{RNA}$, and prevents their functioning in protein synthesis (Erdös \& Ullman, 1959; Spotts \& Stanier, 1961; Flaks, Cox \& White, 1962; Flaks et al. 1962; Speyer, Lengyel \& Basilio, 1962; Mager, Benedict \& Artman, 1962; Davies, 1964). It has also been shown that in normal exponentially growing cultures of bacteria the rate of protein synthesis per (functioning) ribosome is constant, and that changes in growth rates are reflected by changes in ribosome content per cell (Schaechter, Maaløe \& Kjeldgaard, 1958). If one assumes that changes in growth rates could be brought about by changes in content of functioning ribosomes, then exponential growth of dihydrostreptomycin-treated populations at rates equal to $x \%$ of those of controls could mean that the bacteria were multiplying with $x \%$ of their ribosomes functioning. If this were the case, the inhibition of growth rate should be proportional to the degree of saturation of the inhibitory sites. As $y \%$ of such populations later cease to grow, and must therefore have intracellular drug concentrations which will eventually inactivate all their ribosomes, it would follow that the total intracellular antibiotic concentrations of these bacteria must be larger than those necessary to saturate the inhibitory sites initially. We thus conclude that intracellular dihydrostreptomycin (and presumably streptomycin) can be in two phases: an inhibitory fraction at the sites of inhibition (presumably the ribosomes) and a non-inhibitory 'pool' fraction from which the drug could be transferred to inhibitory sites when these become available. The sizes of such pools are assumed to differ in different individuals of treated populations. It is implicit in this hypothesis that there cannot be free interchange between 'pool' and inhibitory sites. The statement by Hancock (1962b) that 'death of the organisms does not appear to follow inevitably the attainment of a certain intracellular concentration of streptomycin, since at lower streptomycin concentrations the organisms were killed after a smaller amount of streptomycin had been taken up and vice versa' already led us to conjecture that the intracellular antibiotic might be heterogenously distributed, and that the amounts in the different phases might be governed by independent factors. Spotts (1962) showed that intracellular streptomycin accumulated by a streptomycin-dependent strain of Escherichia coli consisted of a 'loosely held pool, extractable with hot water, and a firmly-bound fraction, extractable only by hot perchloric acid'. On the other hand, attempts at differential extraction of intracellular ${ }^{14} \mathrm{C}$-streptomycin by Hancock $(1962 b)$ gave no clear-cut results. It seems likely that the existence and size of such 'pools' might vary with the organism and conditions of growth (Kogut \& Lightbown, 1964a).

In our experiments, then, degree of inhibition of growth rates (which are population means) would reflect the degree of saturation of the inhibitory sites at the time of removal of extracellular dihydrostreptomycin, whilst the different percentages of bacteria in the populations which eventually ceased to grow would reflect the different distributions of total intracellular concentrations (and hence pool sizes) of dihydrostreptomycin within these populations. Thus the $y \%$ of the treated populations which eventually ceased to grow would be those whose 'pools' were sufficiently large and stable to continue to transfer antibiotic for combination with 'free' ribosomes as these became available. The rest of the populations, whose 
growth rates eventually recover, would be those whose 'pools' were exhausted before all ribosomes had been inactivated. The fact that the onset of recovery in growth rates does not depend on a given amount of cell synthesis, but occurs after smaller amounts of synthesis with increasing inhibition of growth rate, would suggest that synthesis of ribosomes is uninhibited in the presence of intracellular dihydrostreptomycin as long as growth inhibition is not complete. Such continued ribosome synthesis could then lead to recovery of growth when all intracellular antibiotic had been bound to existing ribosomes and had become unavailable for further combination with new ribosomes. This view implies that active antibiotic cannot be regenerated from the inhibitory 'ribosome-dihydrostreptomycin complex' and hence cannot re-enter the 'pool'.

Whilst this paper was in preparation, Hurwitz, Doppel \& Rosano (1964) reported on the effects of duration of exposure to various concentrations of streptomycin on viability and protein synthesizing ability of Mycobacterium fortuitum. Their data on ${ }^{14} \mathrm{C}$-leucine incorporation, as a measure of protein synthesis, provide a striking parallel to ours on growth, measured by extinction, for the eventual recovery of normal rates after removal of extracellular antibiotic.

We wish to express our thanks to Mr M. Wilkins for able and conscientious technical assistance.

\section{REFERENCES}

BREtz, H. W. (1962). A simple method for estimating slide culture survival. J. Bact. 84, 1115.

Davies, J. E. (1964). Studies on the ribosomes of streptomycin-sensitive and resistant strains of Escherichia coli. Proc. natn. Acad. Sci. U.S.A. 51, 659.

Dubin, D. T. \& DAvis, B. D. (1962). The streptomycin-triggered depolymerization of ribonucleic acid in Escherichia coli. Biochem. biophys. Acta, 55, 793.

Erdös, T. \& UllmanN, A. (1959). Effect of streptomycin on the incorporation of aminoacids labelled with carbon-14 into ribonucleic acid and protein in a cell-free system of Mycobacterium. Nature, Lond., 183, 618.

FuAKs, J. G., Cox, E. C. \& WhITE, J. R. (1962). Inhibition of polypeptide synthesis by streptomycin. Biochem. Biophys. Res. Commun. 7, 385.

Flaks, J. G., Cox, E. C., Whitting, M. L. \& White, J. R. (1962). Polypeptide synthesis with ribosomes from streptomycin-resistant and dependent Escherichia coli. Biochem. Biophys. Res. Commun. 7, 390.

Hancock, R. (1962a). Uptake of ${ }^{14} \mathrm{C}$-streptomycin by some microorganisms and its relation to their sensitivity. J. gen. Microbiol. 28, 493.

HaNcock, R. (1962b). Uptake of ${ }^{14} \mathrm{C}$-streptomycin by Bacillus megaterium. J. gen. Microbiol. 28, 503.

Harris, N. D. \& Whitefield, M. (1963). A lethal effect on damaged Escherichia coli associated with the counting technique. Nature, Lond., 200, 606.

Hurwitz, C., Landau, J. V. \& Doppel, H. W. (1962). Effect of exposure of Escherichia coli to streptomycin on ability to undergo cell division. J. Bact. 84, 1116.

Hurwitz, C. \& Rosano, C. L. (1962). Accumulation of label from ${ }^{14} \mathrm{C}$-streptomycin by Escherichia coli. J. Bact. 83, 1193.

Hurwitz, C., Dopper, H. W. \& Rosano, C. L. (1964). Correlation of the in vivo action of streptomycin on survival and on protein synthesis by Mycobacterium fortuitum. J. gen. Microbiol. 35, 159.

Kogut, M. \& Lightbown, J. W. (1963). Streptomycin action and aerobiosis. Biochem. J. 89, 18 P. 
Kogut, M. \& Lightbown, J. W. (1964a). The mode of action of streptomycin. In Experimental Chemotherapy, vol. 3. Ed. by R. J. Schnitzer \& F. Hawking. New York: Academic Press Inc.

KoGUT, M.\& LightBown, J. W.(1964b). Growth of dihydrostreptomycin-treated Escherichia coli after removal of extracellular antibiotic. J. gen. Microbiol. 34, x.

Kogut, M., Lightbown, J. W. \& Isaacson, P. (1965). Streptomycin action and anaerobiosis. J. gen. Microbiol. 39, 155.

Litwak, G. \& Pramer, D. (1957). Absorption of antibiotics by plant cells. III. Kinetics of streptomycin uptake. Arch. Biochem. Biophys. 68, 396.

Mager, J., Benedict, M. \& Artman, M. (1962). A common site of action for polyamines and streptomycin. Biochim. biophys. Acta, 62, 202.

Miles, A. A. \& Misra, S. A. (1938). The estimation of the bactericidal power of blood. J. Hyg., Camb. 38, 732.

Norris, K. P. \& Powell, E. O. (1961). Improvements in determining total counts of bacteria. J. R. Microscop. Soc. 80, 107.

Postage, J. R., Crumpton, J. E. \& Hunter, J. R. (1961). The measurement of bacterial viability by slide culture. J. gen. Microbiol. 24, 15.

Pramer, D. (1956). Absorption of antibiotics by plant cells. II. Streptomycin. Arch. Biochem. Biophys. 62, 265.

Schaechter, M., Maaløe, O. \& KJeldgaard, N. O. (1958). Dependency on medium and temperature of cell size and chemical composition during balanced growth of Salmonella typhimurium. J. gen. Microbiol. 19, 592.

Speyer, J. F., Lengyel, P. \& Basilio, C. (1962). Ribosomal localization of streptomycin sensitivity. Proc. natn. Acad. Sci. U.S.A. 48, 684.

Spotts, C. R. (1962). Physiological and biochemical studies on streptomycin-dependence in Escherichia coli. J. gen. Microbiol. 28, 347.

Spotts, C. R. \& Stanier, R. Y. (1961). Mechanism of streptomycin action on bacteria: a unitary hypothesis. Nature, Lond., 192, 633.

Szybalski, W. \& MaShima, S. (1959). Uptake of streptomycin by sensitive, resistant and dependent bacteria. Biochem. Biophys. Res. Commun. 1, 249. 